\title{
Geodesic Reduction via Frame Bundle Geometry
}

Ajit BHAND

Department of Mathematics, University of Oklahoma, Norman, OK, USA

E-mail: abhand@math.ou.edu

Received October 12, 2009, in final form February 18, 2010; Published online February 22, 2010

doi:10.3842/SIGMA.2010.020

\begin{abstract}
A manifold with an arbitrary affine connection is considered and the geodesic spray associated with the connection is studied in the presence of a Lie group action. In particular, results are obtained that provide insight into the structure of the reduced dynamics associated with the given invariant affine connection. The geometry of the frame bundle of the given manifold is used to provide an intrinsic description of the geodesic spray. A fundamental relationship between the geodesic spray, the tangent lift and the vertical lift of the symmetric product is obtained, which provides a key to understanding reduction in this formulation.
\end{abstract}

Key words: affine connection; geodesic spray; reduction; linear frame bundle

2010 Mathematics Subject Classification: 53B05; 53C05; 53C22; 58D19

\section{Introduction}

The geometry of systems with symmetry has been an active area of research in the last several years. The study of manifolds with certain special geometric structure invariant under a Lie group action leads to what is known as reduction theory. Such questions arise in, for example, geometric mechanics. In this framework, the presence of symmetry allows the dynamics on a manifold to be studied on a lower dimensional manifold. In mechanics, there are at least three different ways of describing dynamics on a manifold, corresponding to the Lagrangian, Hamiltonian and affine connection formulations respectively. While the reduction theory for Lagrangian and Hamiltonian systems has been well developed (see [1, 9, 10, 20]), these results have been obtained by using variational analysis and symplectic geometry respectively. The main reason behind following this approach is the fact that the dynamics for such systems arises from variational principles which are manifested by symplectic structures in the Hamiltonian framework. However, when the dynamics on a manifold are given in terms of the geodesic equation of an affine connection, we cannot use variational analysis unless additional structure, such as a metric, is provided. Mechanical systems for which the dynamics are given by the geodesics of an affine connection that is not Levi-Civita include systems subjected to velocity constraints (see, for example, [17, 18] and Section 5.4).

We consider an arbitrary affine connection on a manifold invariant under the action of a Lie group and provide results that enable us to decompose the reduced geodesic spray corresponding to the affine connection using tools from differential geometry only. In other words, we do not use variational methods. In arriving at our results, we come to a deeper understanding of the geometry of bundle of linear frames and its relationship with the geometry of the tangent bundle of the given manifold.

The setup we consider is the following. Let $M$ be a manifold and $G$ a Lie group which acts on $M$ in such a manner that $M$ is the total space of a principal bundle over $M / G$. The Lie group $G$ also acts on the bundle $L(M)$ of linear frames over $M$ via the lifted action. It is known that there is a one-to-one correspondence between principal connections on $L(M)$ and affine 
connections on $M$ [15]. Let $\omega$ be a $G$-invariant principal connection on $L(M)$ with $\nabla$ the corresponding affine connection on $M$. The geodesic spray $Z$ corresponding to $\nabla$ is a second-order vector field on the tangent bundle $T M$ with the property that the projection of its integral curves correspond to geodesics on $M$. Thus, to understand how the geodesics evolves under symmetry, $Z$ is the appropriate object to study. Since additional structure is not available, we exploit the geometry of the linear frame bundle in order to fully understand the meaning of the geodesic spray (which is classically defined in local coordinates). The first significant step in this direction is to provide an intrinsic definition of the geodesic spray that uses frame bundle geometry. We are able to provide such a definition.

Moving ahead, we give a new interpretation of the geodesic invariance of a distribution on the manifold $M$ using frame bundle geometry and provide a new proof of a characterization due to Lewis [17] using the symmetric product.

Next, we turn our attention to understanding the reduced geodesic spray of a given connection. Our main idea is that it is possible to study reduction using only geometric data and without variational analysis. To our knowledge, the proposed approach of using frame bundle geometry to study reduction is new. Even though the reduction method is discussed in the context of the geodesic spray, it can be applied to a general invariant second-order vector field.

The reduction procedure presented in this paper is based on the author's thesis [3]. Bullo and Lewis did some preliminary work in this direction [6] and recently, Crampin and Mestdag [13] have presented an approach which is similar in spirit to ours. They consider reduction and reconstruction of general second-order systems and provide a decomposition of the reduced system into three parts.

The definition of the geodesic spray using frame bundle geometry, provided in Section 3, enables us to find a formula relating the geodesic spray and the vertical and complete lifts. In Section 4 we present our reduction methodology along with the main result (Theorem 4.8) which provides a new coordinate-free way of decomposing the reduced geodesic spray in terms of objects defined on the reduced space. Finally, in Section 5 we provide a geometric interpretation of the result in the Riemannian case and discuss some avenues for future work.

\section{Linear connections}

In this section we review some concepts relevant to our investigation and establish notation to be used throughout the paper. Let $M$ be an $n$-dimensional smooth manifold and $L(M)(M, G L(n ; \mathbb{R}))$ the bundle of linear frames with total space $L(M)$, base space $M$, structure group $G L(n ; \mathbb{R})$ and canonical projection $\pi_{M}$. We denote the (right) action of $G L(n ; \mathbb{R})$ by $\Phi: L(M) \times G L(n ; \mathbb{R}) \rightarrow$ $L(M)$. For fixed $a \in G L(n ; \mathbb{R})$, this action induces a map $\Phi_{a}: L(M) \rightarrow L(M)$ given by $\Phi_{a}(u)=\Phi(u, a)$. Recall that a linear frame $u$ at $x \in M$ is an ordered basis $\left(X_{1}, X_{2}, \ldots, X_{n}\right)$ of the tangent space $T_{x} M$. If $a=\left(a_{j}^{i}\right) \in G L(n ; \mathbb{R})$ and $u=\left(X_{1}, X_{2}, \ldots, X_{n}\right)$, then $u a:=\Phi_{a}(u)$ is the linear frame $\left(Y_{1}, Y_{2}, \ldots, Y_{n}\right)$ at $x$ defined by $Y_{i}=\sum_{j=1}^{n} a_{i}^{j} X_{j}$. Equivalently, if $\left(e_{1}, e_{2}, \ldots, e_{n}\right)$ is a standard basis for $\mathbb{R}^{n}$, a frame $u=\left(X_{1}, X_{2}, \ldots, X_{n}\right)$ at $x$ can also be defined as a linear isomorphism $u: \mathbb{R}^{n} \rightarrow T_{x} M$ given by $u e_{i}=X_{i}$. In other words, if $\xi=\sum_{i=1}^{n} \xi^{i} e_{i} \in \mathbb{R}^{n}$, then $u \xi=\sum_{i=1}^{n} \xi^{i} X_{i}$. This is the notion of a linear frame that we will use throughout the paper. The infinitesimal generator corresponding to an element $A \in \mathfrak{g l}(n, \mathbb{R})$ of the Lie algebra will be denoted by $A_{L(M)}$. The canonical form $\theta$ of $L(M)$ is a one-form on $L(M)$ defined by $\theta\left(X_{u}\right)=u^{-1}\left(\pi_{M}\left(X_{u}\right)\right), X_{u} \in T_{u} L(M)$.

A principal connection $\omega$ in the bundle $L(M)(M, G L(n ; \mathbb{R}))$ of linear frames over $M$ is called a linear connection on $M$. The associated horizontal subbundle is denoted by $H L(M)$. The tangent bundle of $M$, denoted by $\tau_{M}: T M \rightarrow M$, is a bundle associated with $L(M)(M, G L(n ; \mathbb{R}))[15]$. 
Given a linear connection $\omega$ on $M$, for each $\xi \in \mathbb{R}^{n}$, the standard horizontal vector field corresponding to $\xi$, denoted by $B(\xi): L(M) \rightarrow T L(M)$, is defined as follows. For each $u \in L(M)$, the vector $B(\xi)_{u}$ is the unique horizontal vector at $u$ with the property that $T_{u} \pi_{M}\left(B(\xi)_{u}\right)=u \xi$.

We consider an arbitrary principal bundle $P(M, G)$ with total space $P$, base space $M$ and structure group $G$. The canonical vertical bundle will be denoted by $V P$.

Given a principal fiber bundle $P(M, G)$ and a representation $\rho$ of $G$ on a finite-dimensional vector space $V$, a pseudotensorial $r$-form of type $(\rho, V)$ on $P$ is a $V$-valued $r$-form $\varphi$ on $P$ such that

$$
\Phi_{g}^{*} \varphi=\rho\left(g^{-1}\right) \cdot \varphi, \quad g \in G,
$$

where $\Phi$ is the action of $G$ on $P$. A pseudotensorial $r$-form $\varphi$ of type $(\rho, V)$ is called a tensorial $r$-form if it is horizontal in the sense that $\varphi\left(X_{1}, \ldots, X_{r}\right)=0$ whenever $X_{i}$ is vertical for at least one $i \in\{1, \ldots, r\}$. A connection one-form $\omega$ on a principal bundle $P(M, G)$ is a pseudotensorial one-form of type $(\operatorname{Ad}(G), \mathfrak{g})$, where $\operatorname{Ad}(G)$ is the adjoint representation of $G$ on $\mathfrak{g}$. The following result characterizing the set of all principal connections on $P$ can be readily proved.

Proposition 2.1. Let $\omega$ be a principal connection one-form on a principal bundle $P(M, G)$ and let $\alpha$ be a tensorial one-form of type $(\operatorname{Ad}(G), \mathfrak{g})$ on $P$. Then $\bar{\omega}:=\omega+\alpha$ defines a new principal connection on $P$. Conversely, given any two principal connection forms $\omega$ and $\bar{\omega}$ respectively, the object $\alpha:=\bar{\omega}-\omega$ is a tensorial one-form of type $(\operatorname{Ad}(G), \mathfrak{g})$ on $P$.

In other words, the space of principal connections is an affine space modeled on the vector space of tensorial one-forms of type $(\operatorname{Ad}(G), \mathfrak{g})$.

To each vector field $Y$ on $M$ we can associate a function $f_{Y}: L(M) \rightarrow \mathbb{R}$ as follows. For $u \in L(M)$, we have $f_{Y}(u)=u^{-1}\left(Y\left(\pi_{M}(u)\right)\right)$. The following result provides a correspondence between tensorial one-forms on $L(M)$ and $(1,2)$ tensor fields on $M$.

Proposition 2.2. There is a one-to-one correspondence between tensorial one-forms of type $(\operatorname{Ad}(G L(n ; \mathbb{R})), \mathfrak{g l}(n ; \mathbb{R}))$ on $L(M)$ and $(1,2)$ tensor fields on $M$.

Proof. Since the tangent bundle $\tau_{M}: T M \rightarrow M$ is the bundle associated with $L(M)$ with standard fiber $\mathbb{R}^{n}$, for each $(1,2)$ tensor field $S$ on $M$, and $u \in L(M)$, we can define a map $\alpha_{S}: T L(M) \rightarrow \mathfrak{g l}(n ; \mathbb{R})$ as follows. Let $\widetilde{X} \in T_{u} L(M)$, for $u \in L(M)$ and let $\eta \in \mathbb{R}^{n}$. Then

$$
u\left(\alpha_{S}(u)(\widetilde{X}) \eta\right)=S\left(T_{u} \pi_{M}(\widetilde{X}), u \eta\right) .
$$

Since $\alpha_{S}(\widetilde{X}) \in \mathfrak{g l}(n ; \mathbb{R})$, the product $\alpha(\widetilde{X}) \eta \in \mathbb{R}^{n}$. We now show that $\alpha_{S}$ is a tensorial form of type $(\operatorname{Ad}(G L(n ; \mathbb{R})), \mathfrak{g l}(n ; \mathbb{R}))$. For $a \in G L(n ; \mathbb{R})$, let $\widetilde{Y}=T_{u} \Phi_{a} \widetilde{X} \in T_{u a} L(M)$. Then, using the definition (2.1), we get

$$
(u a)\left(\alpha_{S}(u a)(\tilde{Y}) \eta\right)=S\left(T_{u a} \pi_{M}(\tilde{Y}), u a \eta\right)=u\left(\alpha_{S}(u)(\tilde{X})(a \eta)\right)
$$

from which we get

$$
\alpha_{S}(u a)(\widetilde{Y})=a^{-1} \alpha_{S}(u)(\widetilde{X}) a,
$$

which means that $\alpha_{S}$ is pseudotensorial. Next, if $\widetilde{X} \in V_{u} L(M)$, it is easy to see that $\alpha_{S}(\tilde{X})=0$, which shows that $\alpha_{S}$ is tensorial.

Conversely, given a tensorial one-form $\alpha: T P \rightarrow \mathfrak{g l}(n ; \mathbb{R})$, we can define a $(1,2)$ tensor field $S_{\alpha}$ as follows:

$$
S_{\alpha}(X, Y)=u\left(\alpha(\tilde{X}) f_{Y}(u)\right), \quad X, Y \in T_{x} M, \quad \pi_{M}(u)=x,
$$

where $\widetilde{X}_{u} \in T_{u} L(M)$ has the property that $T_{u} \pi_{M}(\widetilde{X})=X$. Since $\alpha$ is tensorial, $S_{\alpha}$ is welldefined. 
Corollary 2.3. Let $\omega$ and $\bar{\omega}$ be linear connections of $M$ and let $\nabla$ and $\bar{\nabla}$, respectively, be the corresponding covariant derivatives. If $\alpha=\bar{\omega}-\omega$ then, for vector fields $X$ and $Y$ on $M$, we have

$$
\bar{\nabla}_{X} Y=\nabla_{X} Y+S_{\alpha}(X, Y)
$$

where $S_{\alpha}$ is the unique $(1,2)$ tensor field on $M$ corresponding to $\alpha$.

This result, therefore, characterizes the set of all affine connections on the manifold $M$.

\section{The geodesic spray of an affine connection}

In this section we study the geodesic spray associated with a given affine connection. This object is typically defined in terms of local coordinates and here we provide an intrinsic definition using the geometry of the linear frame bundle. Given a linear connection $\omega$ on $M$, for fixed $\xi \in \mathbb{R}^{n}$, let $\Phi_{\xi}: L(M) \rightarrow T M$ be the association map given by $\Phi_{\xi}(u)=u \xi$. We define a (second-order) vector field $Z: T M \rightarrow T T M$ called the geodesic spray as follows:

$$
Z(v)=T_{u} \Phi_{\xi}\left(B(\xi)_{u}\right), \quad v \in T M
$$

where $u \in L_{\tau_{M}(v)}(M)$ and $\xi \in \mathbb{R}^{n}$ are such that $u \xi=v$, and $B(\xi)$ is the standard horizontal vector field corresponding to $\xi$ for the linear connection $\omega$ associated with $\nabla$. We have the following result.

Proposition 3.1. The map $Z$ defined in (3.1) is a second-order vector field on TM. The coordinate expression for $Z$, in terms of the canonical tangent bundle coordinates $\left(x^{i}, v^{i}\right)$ is given by

$$
Z=v^{i} \frac{\partial}{\partial x^{i}}-\Gamma_{j k}^{i} v^{j} v^{k} \frac{\partial}{\partial v^{i}}
$$

Proof. We first show that $Z$ as given by (3.1) is well-defined. The canonical projection on the tangent bundle is denoted by $\tau_{M}: T M \rightarrow M$. For a given $v \in T M$, we write $x:=\tau_{M}(v)$. Suppose that $u^{\prime} \in L_{x}(M)$ and $\xi^{\prime} \in \mathbb{R}^{n}$ are such that $u^{\prime} \xi^{\prime}=v=u \xi$. Then, we must have $u^{\prime}=u a$ for some $a \in G L(n ; \mathbb{R})$. Consequently, $\xi^{\prime}=a^{-1} \xi$. We compute

$$
T_{u a} \Phi_{a^{-1} \xi}\left(B\left(a^{-1} \xi\right)_{u a}\right)=T_{u a} \Phi_{a^{-1} \xi} T_{u} R_{a}\left(B(\xi)_{u}\right)=T_{u}\left(\Phi_{a^{-1} \xi} \circ R_{a}\right) B(\xi)_{u}=T_{u} \Phi_{\xi}\left(B(\xi)_{u}\right),
$$

where the first equality follows from the properties of a standard horizontal vector field. Let us now show that $Z$ is a second-order vector field. We have

$$
T \tau_{M}(Z(v))=T \tau_{M}\left(T_{u} \Phi_{\xi} B(\xi)_{u}\right)=T_{u}\left(\tau_{M} \circ \Phi_{\xi}\right) B(\xi)_{u}=T_{u}\left(\pi_{M}\right)\left(B(\xi)_{u}\right)=u \xi=v
$$

as desired.

It now remains to be shown that the coordinate representation of $Z$ is as given in (3.2), but this follow directly from the coordinate representation of $B(\xi)$.

\subsection{Tangent and vertical lifts}

If $X$ is a vector field on $M$ we can define a unique vector field $\widetilde{X}$ on $L(M)$ corresponding to $X$ as follows. Let $\phi_{t}^{X}$ be the flow of $X$. The tangent lift $X^{T}$ is a vector field on $T M$ defined by

$$
X^{T}\left(v_{x}\right)=\left.\frac{d}{d t}\right|_{t=0} T \phi_{t}^{X}\left(v_{x}\right)
$$


Let $u \in L_{x}(M)$ and $\xi \in \mathbb{R}^{n}$ be such that $u \xi=v_{x}$. For $\xi$ fixed, let $\Phi_{\xi}: L(M) \rightarrow T M$ be the association map. The flow of $X^{T}$ defines a curve $u_{t}$ in $L(M)$ by $u_{t}=T_{x} \phi_{t}^{X} \cdot u$. That is,

$$
\Phi_{\xi} u_{t}=\left(T_{x} \phi_{t}^{X} \circ \Phi_{\xi}\right) u .
$$

The map $\widetilde{\Phi}_{t}(u)=u_{t}$ defines a flow on $L(M)$. The corresponding vector field is called the natural lift $\widetilde{X}$ of $X$ onto $L(M)$. Thus, we have

$$
X^{T}\left(v_{x}\right)=T_{u} \Phi_{\xi} \tilde{X}(u)
$$

Given $v_{x}, w_{x} \in T_{x} M$, the vertical lift of $w$ at $v$ is defined by

$$
\operatorname{vlft}_{v_{x}}\left(w_{x}\right)=\left.\frac{d}{d t}\right|_{t=0}\left(v_{x}+t w_{x}\right)
$$

\subsection{Decomposition of the geodesic spray}

The following result provides an explicit relationship between $\nabla$ and the connection one-form $\omega$ of the corresponding linear connection.

Proposition 3.2. Let $M$ be a manifold with a connection $\nabla$ with the corresponding linear connection one-form $\omega$. Given vector fields $X$ and $Y$ on $M$, let $\tilde{X}$ be the natural lift of $X$ onto $L(M)$, and $f_{Y}: L(M) \rightarrow \mathbb{R}^{n}$ the function associated with $Y$. Then,

$$
\nabla_{X} Y(x)=[X, Y](x)+u\left(\omega(\tilde{X}(u)) f_{Y}(u)\right), \quad \pi_{M}(u)=x .
$$

Proof. Let us first verify that the right-hand side of (3.3) is independent of the choice of $u \in L_{x}(M)$. For $a \in G L(n ; \mathbb{R})$, we compute

$$
\begin{aligned}
(u a)\left(\omega\left(\widetilde{X}_{u a}\right) f_{Y}(u a)\right) & =(u a)\left(\omega\left(T_{u} \Phi_{a} \widetilde{X}_{u}\right) a^{-1} f_{Y}(u)\right) \\
& =(u a)\left(a^{-1} \omega\left(\widetilde{X}_{u}\right) a\left(a^{-1} f_{Y}(u)\right)\right)=u\left(\omega\left(\widetilde{X}_{u}\right) f_{Y}(u)\right) .
\end{aligned}
$$

We shall now prove that

$$
\mathscr{L}_{X^{h}} f_{Y}(u)=u^{-1}([X, Y](x))+\omega(\widetilde{X}(u)) f_{Y}(u) .
$$

Notice that $\operatorname{hor}(\tilde{X})=X^{h}$ since both are horizontal vector fields on $L(M)$ projecting to $X$. In other words,

$$
X^{h}=\tilde{X}-(\omega(\tilde{X}))_{L(M)},
$$

where $\omega(\widetilde{X})_{L(M)}$ is the vertical vector field on $L(M)$ given by

$$
u \mapsto \omega(\widetilde{X}(u))_{L(M)}(u) .
$$

Therefore,

$$
\mathscr{L}_{X^{h}} f_{Y}(u)=\mathscr{L}_{\tilde{X}} f_{Y}(u)-\mathscr{L}_{\omega(\widetilde{X})_{L(M)}} f_{Y}(u)
$$

The flow of $\tilde{X}$ is $\Phi_{t}^{\widetilde{X}}(u)=T_{x} \Phi_{t}^{X} \cdot u$, where $\Phi_{t}^{X}$ is the flow of $X$ and $x=\pi_{M}(u)$. The first term on the right-hand side of (3.4) is

$$
\begin{aligned}
\mathscr{L}_{\tilde{X}} f_{Y}(u) & =\left.\frac{d}{d t} f_{Y}\left(T_{x} \Phi_{t}^{X} \cdot u\right)\right|_{t=0}=\left.\frac{d}{d t}\left(u^{-1} T_{\Phi_{t}^{X}(x)} \Phi_{-t}^{X}\right)\left(Y\left(\Phi_{t}^{X}(x)\right)\right)\right|_{t=0} \\
& =\left.\frac{d}{d t} u^{-1} \Phi_{t}^{x *} Y(x)\right|_{t=0}=u^{-1}[X, Y](x) .
\end{aligned}
$$


We next compute the second term on the right-hand side of (3.4)

$$
\begin{aligned}
\mathscr{L}_{\omega(\tilde{X})_{L(M)}} f_{Y}(u) & =\left.\frac{d}{d t} f_{Y}(u \exp (t \omega(\widetilde{X}(u))))\right|_{t=0}=\left.\frac{d}{d t}\left(\exp (-t \omega(\widetilde{X}(u))) u^{-1} Y(x)\right)\right|_{t=0} \\
& =-\omega(\widetilde{X}(u)) u^{-1} Y(x)=-\omega(\widetilde{X}(u)) f_{Y}(u),
\end{aligned}
$$

where the second equality above follows from the fact that $f_{X}$ is a pseudotensorial form of degree zero. This completes the proof.

Remark 3.3. It is known that every derivation $\mathscr{D}$ of the tensor algebra of $M$ can be decomposed as

$$
\mathscr{D}=\mathscr{L}_{X}+S
$$

where $X$ is a vector field on $M$ and $S$ is a $(1,1)$ tensor field on $M$. From the previous result, it follows that the $(1,1)$ tensor field associated with the derivation $\nabla_{X}$ is given by

$$
T_{x} M \ni v \mapsto u\left(\omega(\widetilde{X}(u)) f_{Y_{v}}(u)\right), \quad \pi_{M}(u)=x,
$$

where $Y_{v}$ is any vector field on $M$ with value $v$ at $x$.

We now prove the main result of this section.

Proposition 3.4. Let $v \in T_{x} M$ for some $x \in M$, and $X_{v}$ be an arbitrary vector field that has the value $v$ at $x$. Then,

$$
Z(v)=\left(X_{v}\right)^{T}(v)-\operatorname{vlft}_{v}\left(\nabla_{X_{v}} X_{v}(x)\right) .
$$

Proof. Using local coordinates $x^{i}$ around $x$ in $M$, we write $X_{v}=X_{v}{ }^{i} \frac{\partial}{\partial x^{i}}$. Then,

$$
\left(X_{v}\right)^{T}(v)=v^{i} \frac{\partial}{\partial x^{i}}+v^{j} \frac{\partial X_{v}^{i}}{\partial x^{j}} \frac{\partial}{\partial v^{i}},
$$

and

$$
\nabla_{X_{v}} X_{v}(x)=\left(\frac{\partial X_{v}^{i}}{\partial x^{j}} X_{v}^{j}+\Gamma_{j k}^{i} X_{v}^{j} X_{v}{ }^{k}\right) \frac{\partial}{\partial x^{i}}=\left(\frac{\partial X_{v}^{i}}{\partial x^{j}} v^{j}+\Gamma_{j k}^{i} v^{j} v^{k}\right) \frac{\partial}{\partial x^{i}}
$$

So

$$
\operatorname{vlft}_{v}\left(\nabla_{X_{v}} X_{v}(x)\right)=\left(\frac{\partial X_{v}{ }^{i}}{\partial x^{j}} v^{j}+\Gamma_{j k}^{i} v^{j} v^{k}\right) \frac{\partial}{\partial v^{i}} .
$$

Thus,

$$
\begin{aligned}
\left(X_{v}\right)^{T}(v)-\operatorname{vlft}_{v}\left(\nabla_{X_{v}} X_{v}(x)\right) & =v^{i} \frac{\partial}{\partial x^{i}}+v^{j} \frac{\partial X_{v}^{i}}{\partial x^{j}} \frac{\partial}{\partial v^{i}}-\left(\frac{\partial X_{v}^{i}}{\partial x^{j}} v^{j}+\Gamma_{j k}^{i} v^{j} v^{k}\right) \frac{\partial}{\partial v^{i}} \\
& =v^{i} \frac{\partial}{\partial x^{i}}-\Gamma_{j k}^{i} v^{j} v^{k} \frac{\partial}{\partial v^{i}}=Z(v) .
\end{aligned}
$$

This proves the result. Notice that, even though each of the two terms $X_{v}^{T}(v)$ and $\operatorname{vlft}_{v}\left(\nabla_{X_{v}} X_{v}\right)$ depends on the extension $X_{v}$, the terms that depend on the derivative of $X_{v}$ cancel in the expression for $Z$. 
Alternate proof. Given $v_{x} \in T_{x} M$, let $u \in L_{x} M$ and $\xi \in \mathbb{R}^{n}$ be such that $u \xi=v_{x}$. Let $X_{v}$ be a vector field with value $v_{x}$ at $x$ and $f_{X_{v}}: L(M) \rightarrow \mathbb{R}^{n}$ be the corresponding function on $L(M)$. Then, for each $u \in L(M)$,

$$
\operatorname{hor}\left(\widetilde{X_{v}}(u)\right)=B\left(f_{X_{v}}(u)\right)_{u},
$$

where $\widetilde{X_{v}}$ is the natural lift of $X_{v}$ onto $L(M)$. We have

$$
\begin{aligned}
Z\left(X_{v}(x)\right) & =T_{u} \Phi_{f_{X_{v}}(u)} B\left(f_{X_{v}}(u)\right)_{u}=T_{u} \Phi_{f_{X_{v}}(u)}\left(\tilde{X}(u)-\omega(\widetilde{X}(u))_{L(M)}\right) \\
& =X_{v}^{T}\left(X_{v}(x)\right)-T_{u} \Phi_{f_{X_{v}}(u)}\left(\omega(\widetilde{X}(u))_{L(M)}\right) \\
& =X_{v}^{T}\left(X_{v}(x)\right)-\left.\frac{d}{d t} \Phi_{f_{X_{v}}(u)} u \exp (t \omega(\tilde{X}(u)))\right|_{t=0} \\
& =X_{v}^{T}\left(X_{v}(x)\right)-\operatorname{vlft}_{X_{v}(x)} u\left(\omega(\widetilde{X}(u)) f_{X_{v}}(u)\right) \\
& =X_{v}^{T}\left(X_{v}(x)\right)-\operatorname{vlft}_{X_{v}(x)} \nabla_{X_{v}} X_{v}(x),
\end{aligned}
$$

where we have used Proposition 3.2 in the last step.

\subsection{Geodesic invariance}

We recall the notion of geodesic invariance.

Definition 3.5. A distribution $D$ on a manifold $M$ with an affine connection $\nabla$ is called geodesically invariant if for every geodesic $c:[a, b] \rightarrow M, \dot{c}(a) \in D_{c(a)}$ implies that $\dot{c}(t) \in D_{c(t)}$ for all $t \in[a, b]$.

It turns out that geodesic invariance can be characterized by studying a certain product on the set of vector fields on $M$. Let $M$ be a manifold with a connection $\nabla$. Given vector fields $X, Y \in \Gamma(T M)$, the symmetric product $\langle X: Y\rangle$ is the vector field defined by

$$
\langle X: Y\rangle=\nabla_{X} Y+\nabla_{Y} X .
$$

The following result gives a description of the symmetric product using linear frame bundle geometry.

Proposition 3.6. Let $M$ be a manifold with a connection $\nabla$, let $\omega$ be the associated linear connection and $\theta$ the canonical form on $L(M)$ respectively. If $X$ and $Y$ are vector fields on $M$ and $\widetilde{X}$ and $\widetilde{Y}$ the respective natural lifts on $L(M)$, then

$$
\langle X: Y\rangle(x)=2 u(\operatorname{Sym}(\omega \otimes \theta)(\tilde{X}, \tilde{Y})), \quad \pi_{M}(u)=x .
$$

Proof. It is clear that the right-hand side of (3.7) is independent of the choice of $u \in L_{x}(M)$. By definition, we have $\theta(\widetilde{X}(u))=f_{X}(u)$ and $\theta(\widetilde{Y}(u))=f_{Y}(u)$. We compute

$$
\begin{aligned}
2 u(\operatorname{Sym}(\omega \otimes \theta)(\widetilde{X}(u), \widetilde{Y}(u))) & =u(\omega(\widetilde{X}(u)) \theta(\widetilde{Y}(u))+\omega(\widetilde{Y}(u)) \theta(\widetilde{X}(u))) \\
& =u\left(\omega(\widetilde{X}(u)) f_{Y}(u)+\omega(\widetilde{Y}(u)) f_{X}(u)\right) .
\end{aligned}
$$

The result now follows from Proposition 3.2.

Remark 3.7. The object $\operatorname{sym}(\omega \otimes \theta)$ defines a quadratic form $\Sigma_{u}: T_{u} L(M) \times T_{u} L(M) \rightarrow$ $T_{u} L(M), u \in L(M)$ as follows:

$$
\Sigma_{u}(X, Y)=\widetilde{Z_{X, Y}}(u), \quad X, Y \in T_{u} L(M),
$$

where $\widetilde{Z_{X, Y}}$ is the natural lift onto $L(M)$ of the vector field $Z_{X, Y}$ on $M$ given by

$$
Z_{X, Y}(x)=u(\operatorname{sym}(\omega \otimes \theta)(X, Y)), \quad \pi(u)=x .
$$

This is seen to be well-defined. 
Given a distribution $D$ on $M$, we represent by $\Gamma(D)$ the set of vector fields taking values in $D$. The following result, proved by Lewis [17], provides infinitesimal tests for geodesic invariance and gives the geometric meaning of the symmetric product.

Theorem 3.8 (Lewis). Let $D$ be a distribution on a manifold $M$ with a connection $\nabla$. The following are equivalent:

(i) $D$ is geodesically invariant;

(ii) $\langle X: Y\rangle \in \Gamma(D)$ for every $X, Y \in \Gamma(D)$;

(iii) $\nabla_{X} X \in \Gamma(D)$ for every $X \in \Gamma(D)$.

We give an intrinsic proof of this theorem below. Thus, for geodesically invariant distributions, the symmetric product plays the role that the Lie bracket plays for integrable distributions. We use this result to interpret some terms obtained in the decomposition of the reduced geodesic spray in Section 4.

Now, given a $p$-dimensional distribution $D$ on an $n$-dimensional manifold $M$ with a linear connection, we say that a frame $u \in L_{x}(M)$ is $D$-adapted if $\left.u\right|_{\mathbb{R}^{p}}$ is an isomorphism onto $D_{x}$. Let $L(M, D)$ be the collection of $D$-adapted frames. We observe that $L(M, D)$ is invariant under the subgroup of $G L(n ; \mathbb{R})$ consisting of those automorphisms which leave $\mathbb{R}^{p}$ invariant. It turns out that $L(M, D)$ is a subbundle of $L(M)$ with structure group $H$ given by

$$
H=\left\{A \in G L(n ; \mathbb{R}) \mid A=\left(\begin{array}{ll}
a & b \\
0 & c
\end{array}\right), a \in G L(p ; \mathbb{R}), b \in L\left(\mathbb{R}^{n-p}, \mathbb{R}^{p}\right), c \in G L(n-p ; \mathbb{R})\right\} .
$$

We denote the bundle of $D$-adapted frames by $L(M, D)(M, H)$ and the Lie algebra of $H$ by $\mathfrak{h}$. We have the following result.

Proposition 3.9. The distribution $D$ is geodesically invariant if and only if, for each $\xi \in \mathbb{R}^{p}$, $\left.B(\xi \oplus 0)\right|_{L(M, D)}$ is a vector field on $L(M, D)$.

Proof. We first prove the "if" statement. Suppose that $B(\xi \oplus 0)$ is a vector field on $L(M, D)$ and let $c: \mathbb{R} \rightarrow L(M)$ be its integral curve passing through $\bar{u} \in L(M, D)$. Then, we know that $x(t):=\pi_{M}(c(t))$ is the unique geodesic with the initial condition $\bar{u} \xi \in D$. We must show that $\dot{x}(t) \in D_{x(t)}$ for all $t$. We have

$$
\dot{x}(t)=T \pi_{M}\left(B(\xi \oplus 0)_{c(t)}\right)=c(t)(\xi \oplus 0) .
$$

Since $B(\xi \oplus 0)$ is a vector field on $L(M, D)$, we must have $c(t) \in L(M, D)$ for all $t$. Thus, we have $\dot{x}(t) \in D_{x(t)}$ for all $t$. The "only if" part of the statement can be proved by reversing this argument.

An immediate consequence of this result is the following.

Corollary 3.10. A distribution $D$ is geodesically invariant if and only if the geodesic spray $Z$ is tangent to the submanifold $D$ of $T M$.

We are now in a position to provide a proof of Theorem 3.8 using frame bundle geometry.

Proof of Theorem 3.8. $(i) \Longrightarrow(i i)$ Suppose that $D$ is geodesically invariant, and let $X_{1}, X_{2} \in$ $\Gamma(D)$. Then, we know that the corresponding functions $f_{X_{i}}: L(M, D) \rightarrow \mathbb{R}^{p} \oplus \mathbb{R}^{n-p}, i=1,2$, take values in $\mathbb{R}^{p}$. Also,

$$
\left(X_{i}\right)^{h}(u)=c_{i}^{j} B\left(e_{j} \oplus 0\right)_{u}, \quad u \in L(M, D),
$$


where $c_{i}^{j}$ are functions on $L(M)$ and $\left\{e_{j}\right\}_{j=1, \ldots, p}$ is the standard basis for $\mathbb{R}^{p}$. This is possible since $\left\{B\left(e_{i}\right)\right\}$ form a basis for $H_{u} L(M)$. We have

$$
f_{\left(\nabla_{X_{1}} X_{2}+\nabla_{X_{2}} X_{1}\right)}=\mathscr{L}_{\left(X_{1}\right)^{h}} f_{X_{2}}+\mathscr{L}_{\left(X_{2}\right)^{h}} f_{X_{1}}=c_{1}^{j} \mathscr{L}_{B\left(e_{j} \oplus 0\right)} f_{X_{2}}+c_{2}^{k} \mathscr{L}_{B\left(e_{k} \oplus 0\right)} f_{X_{1}} .
$$

Since $f_{X_{i}}, i=1,2$, are $\mathbb{R}^{p}$-valued functions on $L(M, D)$ and $\left.B\left(e_{j} \oplus 0\right)\right|_{L(M, D)}, j=1, \ldots, p$, are vector fields on $L(M, D)$ because the distribution is assumed to be geodesically invariant, we conclude that the function $f_{\left(\nabla_{X_{1}} X_{2}+\nabla_{X_{2}} X_{1}\right)}: L(M, D) \rightarrow \mathbb{R}^{p} \oplus \mathbb{R}^{n-p}$ takes its values in $\mathbb{R}^{p}$. This proves $(i i)$.

(ii) $\Longrightarrow($ iii $)$ This follows directly from the definition of the symmetric product.

(iii) $\Longrightarrow\left(\right.$ i) Assume that $\nabla_{X} X \in \Gamma(D)$ for every $X \in \Gamma(D)$. This implies that the function $\mathscr{L}_{X^{h}} f_{X}: L(M, D) \rightarrow \mathbb{R}^{p} \oplus \mathbb{R}^{n-p}$ takes values in $\mathbb{R}^{p}$. Once again, we can write $X^{h}=C^{i} B\left(e_{i} \oplus 0\right)$ for some functions $C^{i}$. This implies that $\left.B\left(e_{i} \oplus 0\right)\right|_{L(M, D)}$ must be a vector field on $L(M, D)$.

The above result shows that it is possible to check for geodesic invariance by looking at vector fields $B\left(e_{i} \oplus 0\right)$ on the bundle $L(M, D)$.

Theorem 3.8 and Proposition 3.6 suggest the following:

Corollary 3.11. Let $D$ be a p-dimensional distribution on a manifold $M$ with a linear connection $\omega$. Let $\widetilde{D}$ be the natural lift of $D$ onto $L(M)$ and $L(M, D)(M, H)$ the bundle adapted to $D$. The following statements are equivalent:

(i) $D$ is geodesically invariant;

(ii) $\operatorname{Sym}(\omega \otimes \theta)$ is an $\mathbb{R}^{p}$-valued quadratic form on $\left.\widetilde{D}\right|_{L(M, D)}$;

(iii) $\omega(\widetilde{X}(u)) \in \mathfrak{h}$ for all $\widetilde{X} \in \Gamma(\widetilde{D}), u \in L(M, D)$.

\section{Geodesic reduction}

In this section we consider the following setup. Let $M(M / G, G)$ be a principal fiber bundle with structure group $G$ and let $\nabla$ be a $G$-invariant affine connection on $M$. We study the "reduced" geodesic spray corresponding to $\nabla$ and use a principal connection to decompose it into various components. These components correspond to geometric objects defined on $M / G$.

\subsection{Invariant affine connections}

Let $M(M / G, G)$ be a principal fiber bundle with a $G$-invariant affine connection $\nabla$ on $M$. Choose a principal connection $A$ on $M(M / G, G)$. With this data, we can define an affine connection $\nabla^{A}$ on $M / G$ as follows.

Proposition 4.1. Let $\nabla$ and $A$ be as above. Given vector fields $X$ and $Y \in \Gamma(T(M / G))$ on $M / G$, the map $\nabla^{A}: \Gamma(T(M / G)) \times \Gamma(T(M / G)) \rightarrow \Gamma(T(M / G))$ defined by

$$
\nabla_{X}^{A} Y(x)=T \pi_{M / G} \nabla_{X^{h}} Y^{h}(q), \quad q \in M, \quad \pi_{M / G}(q)=x \in M / G
$$

is an affine connection on $M / G$.

Proof. It is easy to see that $\nabla^{A}$ is well-defined. Given a smooth function $f: M / G \rightarrow \mathbb{R}$, we define the lift $\tilde{f}: M \rightarrow \mathbb{R}$ by $\tilde{f}=\pi_{M / G}^{*} f$. We have

$$
\begin{aligned}
\nabla_{X}^{A}(f Y)(x) & =T \pi_{M / G} \nabla_{X^{h}}\left(\tilde{f} Y^{h}\right)(q)=T \pi_{M / G}\left(\tilde{f}(q) \nabla_{X^{h}} Y^{h}(q)+\left(\mathscr{L}_{x^{h}} \tilde{f}\right)(q) \cdot Y^{h}(q)\right) \\
& =f(x) \nabla_{X}^{A} Y(x)+\left(\mathscr{L}_{X} f\right)(x) \cdot Y(x),
\end{aligned}
$$

where the last part follows since the integral curves of $X^{h}$ project to integral curves of $X$. The fact that $\nabla^{A}$ satisfies all the other properties of an affine connection is easily verified. 


\subsection{The reduced geodesic spray and its decomposition}

In this section we carry out the reduction of the geodesic spray corresponding to an invariant affine connections. Let $M(M / G, G)$ be a principal fiber bundle with total space $M$ and structure group $G$. We denote the action of $G$ on $M$ by $\Phi: G \times M \rightarrow M$ and its tangent lift by $\Phi^{T}: G \times T M \rightarrow T M$. The tangent bundle projection is denoted by $\tau_{M}: T M \rightarrow M$. We can define a map $\left[\tau_{M}\right]_{G}: T M / G \rightarrow M / G$ as follows:

$$
\left[\tau_{M}\right]_{G}\left([v]_{G}\right)=\left[\tau_{M}(v)\right]_{G}, \quad[v]_{G} \in T M / G .
$$

It is easy to see that $\left[\tau_{M}\right]_{G}: T M / G \rightarrow M / G$ is a vector bundle. The adjoint bundle with $\mathfrak{g}$ as the fiber and $M / G$ as the base space will be represented by $\tilde{\mathfrak{g}}_{M / G}$. A typical element of $\tilde{\mathfrak{g}}_{M / G}$ will be denoted by $[x, \xi]_{G}$, where $x \in M$ and $\xi \in \mathfrak{g}$. We shall also denote the tangent bundle of $M / G$ by $\tau_{M / G}: T(M / G) \rightarrow M / G$. If $A$ is a principal connection on the bundle $\pi_{M / G}: M \rightarrow M / G$, we can decompose the bundle $T M / G$ into its horizontal and vertical parts [19].

Lemma 4.2. The map $\alpha_{A}: T M / G \rightarrow T(M / G) \oplus \tilde{\mathfrak{g}}_{M / G}$ given by

$$
\alpha_{A}\left(\left[v_{x}\right]_{G}\right)=T \pi_{M / G}\left(v_{x}\right) \oplus\left[x, A\left(v_{x}\right)\right]_{G}, \quad v_{x} \in T_{x} M, \quad x \in M
$$

is a vector bundle isomorphism.

We denote the $\tilde{\mathfrak{g}}$ component of $\alpha_{A}$ by $\rho_{A}: T M / G \rightarrow \tilde{\mathfrak{g}}$. This decomposition of $T M / G$ is $A$-dependent and we write $T M / G \simeq T(M / G) \oplus_{A} \tilde{\mathfrak{g}}_{M / G}$. The lifted action $\Phi^{T}$ makes $T M$ the total space of a principal bundle over $T M / G$ with structure group $G$. We denote the canonical projection by $\pi_{T M / G}: T M \rightarrow T M / G$. Furthermore, $G$ acts on $T T M$ by the tangent lift of $\Phi^{T}$. We denote by $\overline{T \tau_{M}}: T T M / G \rightarrow T M / G$ the map given by

$$
\overline{T \tau_{M}}\left(\left[W_{v_{x}}\right]_{G}\right)=\left[T \tau_{M}\left(W_{v_{x}}\right)\right]_{G}, \quad W_{v_{x}} \in T_{v_{x}} T M .
$$

It is easy to see that this map is well-defined. By Lemma 4.2, a principal connection $\hat{A}$ on $T M(T M / G, G)$ induces an isomorphism between bundles $T T M / G$ and $T(T M / G) \oplus \tilde{\mathfrak{g}}_{T M / G}$ over $T M / G$. Thus, if $A$ and $\hat{A}$ are chosen, we can consider an identification of $T T M / G$ and $T T(M / G) \oplus_{A} T \tilde{\mathfrak{g}}_{M / G} \oplus_{\hat{A}} \tilde{\mathfrak{g}}_{T M / G}$ where we identify $T(T M / G)$ and $T(T(M / G)) \oplus_{A} T \tilde{\mathfrak{g}}$ using the map $T \alpha_{A}$. The following Lemma will be useful in our decomposition of the geodesic spray.

Lemma 4.3. Given a principal connection $A$ on $M(M / G, G)$, the pullback $\hat{A}:=\tau_{M}^{*} A$ is a principal connection on $T M(T M / G, G)$.

The connection $\hat{A}$ has the following useful property.

Corollary 4.4. If $S: T M \rightarrow T T M$ is a second-order vector field, then

$$
\rho_{\hat{A}}\left(\left[S\left(v_{x}\right)\right]_{G}\right)=\left[v_{x}, A\left(v_{x}\right)\right]_{G} \in \tilde{\mathfrak{g}}_{T M / G} .
$$

In other words, if we choose connections $A$ and $\hat{A}$ on $M(M / G, G)$ and $T M(T M / G, G)$ respectively, studying a second-order vector field such as the geodesic spray reduces to studying the $T T(M / G) \oplus T \tilde{\mathfrak{g}}$ components, since the $\tilde{\mathfrak{g}}_{T M / G}$ component is completely determined by $A$ itself. We now define the reduced geodesic spray.

Proposition 4.5. Let $\omega$ be a $G$-invariant linear connection on $L(M)$ and $\nabla$ the corresponding connection on $M$. The map $\bar{Z}: T M / G \rightarrow T T M / G$ given by

$$
\bar{Z}\left(\left[v_{x}\right]_{G}\right)=\left[Z\left(v_{x}\right)\right]_{G}=\left[T_{u} \Phi_{\xi} B(\xi)_{u}\right]_{G}
$$

is well-defined. We call $\bar{Z}$ the reduced geodesic spray. 
Proof. $G$-invariance of $\omega$ implies the invariance of the standard horizontal vector fields. The result now follows from the $G$-equivariance of the association map $\Phi_{\xi}: L(M) \rightarrow T M, \xi \in \mathbb{R}^{n}$.

Now, since $G$ acts on $T T M$ via the lifted action, we can define a map $\overline{T \pi_{T M / G}}: T T M / G \rightarrow$ $T(T(M / G))$ as follows:

$$
\overline{T \pi_{T M / G}}\left[W_{v_{x}}\right]_{G}=T \pi_{T M / G}\left(W_{v_{x}}\right), \quad\left[W_{v_{x}}\right]_{G} \in T T M / G .
$$

This is well-defined since given any $g \in G$, we have $\pi_{T M / G} \circ T \Phi_{g}^{T}=\pi_{T M / G}$. By abuse of notation, we shall use the maps $T \pi_{T M / G}$ and $\overline{T \pi_{T M / G}}$ interchangeably. We have the following result.

Proposition 4.6. Let $S_{Z}: T(M / G) \rightarrow T T(M / G)$ be the map defined by

$$
S_{Z}(\bar{X})=T\left(T \pi_{M / G} \circ \pi_{T M / G}\right) \bar{Z}\left(\left[\bar{X}^{h}(x)\right]_{G}\right), \quad \bar{X} \in T_{[x]_{G}}(M / G),
$$

where $\bar{X}^{h}$ is an invariant horizontal vector field that projects to $\bar{X}$ at $x \in M$. The following statements hold:

(i) $S_{Z}$ is a second-order vector field on $T(M / G)$;

(ii) $S_{Z}(\bar{X})=\bar{X}^{T}(\bar{X})-\operatorname{vlft}_{\bar{X}} T \pi_{M / G}\left(\nabla_{\bar{X}^{h}} \bar{X}^{h}\right)$, where, by abuse of notation, $\bar{X}$ is a vector field on $M / G$ which has a value $\bar{X}$ at $[x]_{G} \in M / G$.

Proof. (i) We compute

$$
\begin{aligned}
T \tau_{M / G} S_{Z}(\bar{X}) & =T\left(\tau_{M / G} \circ T \pi_{M / G}\right) Z\left(\bar{X}^{h}(x)\right) \\
& =T \pi_{M / G} T \tau_{M}\left(Z\left(\bar{X}^{h}(x)\right)\right)=T \pi_{M / G}\left(\bar{X}^{h}(x)\right)=\bar{X} .
\end{aligned}
$$

(ii) Let $\Phi_{t}^{\bar{X}^{h}}$ and $\Phi_{t}^{\bar{X}}$ be the flows of $\bar{X}^{h}$ and $\bar{X}$ respectively. We have

$$
\begin{aligned}
T T \pi_{M / G}\left(\bar{X}^{h}\right)^{T}\left(\bar{X}^{h}(x)\right) & =\left.\frac{d}{d t}\right|_{t=0}\left(T \pi_{M / G} \circ T \Phi_{t}^{X^{h}}\right)\left(\bar{X}^{h}(x)\right) \\
& =\left.\frac{d}{d t}\right|_{t=0} T\left(\pi_{M / G} \circ \Phi_{t}^{X^{h}}\right)\left(\bar{X}^{h}(x)\right) \\
& =\left.\frac{d}{d t}\right|_{t=0}\left(\left.\frac{d}{d s}\right|_{s=0}\left(\pi_{M / G} \circ \Phi_{t}^{X^{h}}\right)\left(\Phi_{s}^{X^{h}}(x)\right)\right) \\
& =\left.\left.\frac{d}{d t}\right|_{t=0} \frac{d}{d s}\right|_{s=0}\left(\pi_{M / G} \circ \Phi_{t+s}^{X^{h}}(x)\right) \\
& =\left.\left.\frac{d}{d t}\right|_{t=0} \frac{d}{d s}\right|_{s=0}\left(\Phi_{t+s}^{X}\left([x]_{G}\right)=\bar{X}^{T}(\bar{X}) .\right.
\end{aligned}
$$

Next, we look at

$$
\begin{aligned}
T T \pi_{M / G} \operatorname{vlft}_{\bar{X}^{h}(x)}\left(\nabla_{\bar{X}^{h}} \bar{X}^{h}(x)\right) & =\left.\frac{d}{d t}\left(t T \pi_{M / G} \nabla_{\bar{X}^{h}} \bar{X}^{h}(x)+T \pi_{M / G} \bar{X}^{h}(x)\right)\right|_{t=0} \\
& =\left.\frac{d}{d t}\left(t T \pi_{M / G} \nabla_{\bar{X}^{h}} \bar{X}^{h}(x)+\bar{X}\left([x]_{G}\right)\right)\right|_{t=0} \\
& =\operatorname{vlft}_{\bar{X}\left([x]_{G}\right)} T \pi_{M / G} \nabla_{\bar{X}^{h}} \bar{X}^{h}(x) .
\end{aligned}
$$

This gives us

$$
S_{Z}(\bar{X})=\bar{X}^{T}(\bar{X})-\operatorname{vlft}_{\bar{X}}\left(T \pi_{M / G} \nabla_{\bar{X}^{h}} \bar{X}^{h}(x)\right) .
$$

The result now following from Proposition 4.1. 
The idea here is that we use principal connections $A$ and $\tau_{M}^{*} A$ on $M(M / G, G)$ and $T M(T M / G, G)$, respectively, to write the reduced geodesic spray corresponding to an invariant linear connection as a map from $T(M / G) \oplus \tilde{\mathfrak{g}}$ to $T T(M / G) \oplus T \tilde{\mathfrak{g}}$. The map $S_{Z}$ gives us one component of this decomposition. From Proposition 3.4, we see that $S_{Z}$ is the geodesic spray of the affine connection $\nabla^{A}$ on $M / G$.

Next, we define a map $P_{Z}: \tilde{\mathfrak{g}} \rightarrow T T(M / G)$ as follows:

$$
P_{Z}\left([x, \xi]_{G}\right)=T T \pi_{M / G} T \pi_{T M / G} \bar{Z}\left(\left[\xi_{L}^{V}(x)\right]_{G}\right),
$$

where $\xi_{L}^{V}$ is the left-invariant vector field on $M$ that satisfies $\xi_{L}^{V}(x)=\xi_{M}(x)$. We must verify that this is well-defined. To see this, notice that $\left[g \cdot x, \operatorname{Ad}_{g} \xi\right]_{G}=[x, \xi]_{G}$. Next, we have

$$
\begin{aligned}
\left(\operatorname{Ad}_{g} \xi\right)_{M}(g \cdot x) & =\left.\frac{d}{d t} \Phi_{\exp \left(\operatorname{Ad}_{g} \xi\right) t}(g \cdot x)\right|_{t=0}=\left.\frac{d}{d t} \Phi\left(g(\exp \xi t) g^{-1}, g \cdot x\right)\right|_{t=0} \\
& =T_{x} \Phi_{g} \xi_{M}(x)=\xi_{L}^{V}(g \cdot x) .
\end{aligned}
$$

Let us denote $\tilde{\xi}:=[x, \xi]_{G}$. Using (3.5), we get

$$
\begin{aligned}
P_{Z}(\tilde{\xi})=T T \pi_{M / G} Z\left(\xi_{L}^{V}(x)\right) & =T T \pi_{M / G}\left(\left(\xi_{L}^{V}\right)^{T}\left(\xi_{L}^{V}(x)\right)\right)-T T \pi_{M / G} \operatorname{vlft}_{\xi_{L}^{V}(x)}\left(\nabla_{\xi_{L}^{V}} \xi_{L}^{V}(x)\right) \\
& =-\operatorname{vlft}_{\mathbf{0}}\left(T \pi_{M / G} \nabla_{\xi_{L}^{V}} \xi_{L}^{V}(x)\right) .
\end{aligned}
$$

We write $\mathscr{S}(\tilde{\xi}, \tilde{\xi})=\left(T \pi_{M / G} \nabla_{\xi_{L}^{V}} \xi_{L}^{V}(x)\right)$. Since $\nabla$ is $G$-invariant, this map is well-defined.

Next, we define $R_{Z}: T(M / G) \rightarrow T \tilde{\mathfrak{g}}$ by

$$
R_{Z}(\bar{X})=T \rho_{A} T \pi_{T M / G} Z\left(\bar{X}^{h}(x)\right) .
$$

Then, using (3.5), we calculate

$$
T \rho_{A} T \pi_{T M / G} Z\left(\bar{X}^{h}(x)\right)=T \rho_{A} T \pi_{T M / G}\left(\left(\bar{X}^{h}\right)^{T}\left(\bar{X}^{h}(x)\right)-\operatorname{vlft}_{\bar{X}^{h}(x)}\left(\nabla_{\bar{X}^{h}} \bar{X}^{h}(x)\right)\right) .
$$

Let us look at the first term on the right-hand side

$$
\begin{aligned}
T \rho_{A} T \pi_{T M / G}\left(\left(\bar{X}^{h}\right)^{T}\left(\bar{X}^{h}(x)\right)\right) & =\left.T \rho_{A} T \pi_{T M / G} \frac{d}{d t}\right|_{t=0} T \Phi_{t}^{\bar{X}^{h}}\left(\bar{X}^{h}(x)\right) \\
& =\left.\frac{d}{d t}\right|_{t=0} \rho_{A}\left(\left[T \Phi_{t}^{\bar{X}^{h}}\left(\bar{X}^{h}(x)\right)\right]_{G}\right)=0,
\end{aligned}
$$

since $T \Phi_{t}^{\bar{X}^{h}}\left(\bar{X}^{h}(x)\right)$ is horizontal and $\rho_{A}$ vanishes on horizontal vectors. Also,

$$
\begin{aligned}
T \rho_{A} T \pi_{T M / G} \operatorname{vlft}_{\bar{X}^{h}(x)} & \left(\nabla_{\bar{X}^{h}} \bar{X}^{h}(x)\right)=\left.\frac{d}{d t}\right|_{t=0} \rho_{A} \pi_{T M / G}\left(t \nabla_{\bar{X}^{h}} \bar{X}^{h}(x)+\bar{X}^{h}(x)\right) \\
& =\left.\frac{d}{d t}\right|_{t=0}\left(t \rho_{A} \circ \pi_{T M / G}\left(\nabla_{\bar{X}^{h}} \bar{X}^{h}(x)\right)+\rho_{A} \circ \pi_{T M / G}\left(\bar{X}^{h}(x)\right)\right) \\
& =\left.\frac{d}{d t}\right|_{t=0}\left(t \rho_{A} \circ \pi_{T M / G}\left(\nabla_{\bar{X}^{h}} \bar{X}^{h}(x)\right)+0\right),
\end{aligned}
$$

and thus we get

$$
R_{Z}(\bar{X})=-\operatorname{vlft}_{0}\left(\rho_{A} \circ \pi_{T M / G}\left(\nabla_{\bar{X}^{h}} \bar{X}^{h}(x)\right)\right) .
$$

If $H M$ is geodesically invariant, then $\nabla_{\bar{X}^{h}} \bar{X}^{h}$ is horizontal, and thus $R_{Z}=0$. 
Finally, we define $U_{Z}: \tilde{\mathfrak{g}} \rightarrow T \tilde{\mathfrak{g}}$ by

$$
U_{Z}(\tilde{\xi})=T \rho_{A} T \pi_{T M / G} Z\left(\xi_{L}^{V}(x)\right),
$$

and a calculation similar to the one performed above shows that

$$
U_{Z}(\tilde{\xi})=-\operatorname{vlft}_{\xi} \rho_{A}\left(\pi_{T M / G}\left(\nabla_{\xi_{L}^{V}} \xi_{L}^{V}(x)\right)\right) .
$$

The following lemma is useful.

Lemma 4.7. The map $\widetilde{\nabla}^{A}: \Gamma(T(M / G)) \times \Gamma(\tilde{\mathfrak{g}}) \rightarrow \Gamma(\tilde{\mathfrak{g}})$ given by

$$
\widetilde{\nabla}_{\bar{X}}^{A} \tilde{\xi}\left([x]_{G}\right)=\rho_{A} \pi_{T M / G}\left(\left\langle\bar{X}^{h}: \xi_{L}^{V}\right\rangle(x)\right), \quad[x]_{G} \in(M / G)
$$

defines a vector bundle connection on the bundle $\tilde{\mathfrak{g}}$.

Proof. Let $f: M / G \rightarrow \mathbb{R}$ be a differentiable function. Define $f^{h}: M \rightarrow \mathbb{R}$ by $f^{h}=\pi_{M / G}^{*} f$. Therefore, $(f \bar{X})^{h}=f^{h} \bar{X}^{h}$. We compute

$$
\begin{aligned}
\widetilde{\nabla}_{f \bar{X}}^{A} \tilde{\xi} & =\rho_{A} \pi_{T M / G}\left(\left\langle f^{h} \bar{X}^{h}: \xi_{L}^{V}\right\rangle\right) \\
& =\rho_{A} \pi_{T M / G}\left(f^{h} \nabla_{\bar{X}^{h}} \xi_{L}^{V}+f^{h} \nabla_{\xi_{L}^{V}} \bar{X}^{h}+\left(\mathscr{L}_{\xi_{L}^{V}} f^{h}\right) \bar{X}^{h}\right)=f \widetilde{\nabla}_{\bar{X}}^{A} \tilde{\xi}
\end{aligned}
$$

since $\left(\mathscr{L}_{\xi_{L}^{V}} f^{h}\right) \bar{X}^{h}=0$. The property $\widetilde{\nabla}_{\bar{X}}^{A} f \tilde{\xi}=f \widetilde{\nabla}_{\bar{X}}^{A} \tilde{\xi}+\left(\mathscr{L}_{\bar{X}} f\right) \tilde{\xi}$ can be proved similarly.

We now state the main result of this section.

Theorem 4.8. Let $Z_{h}: T(M / G) \oplus \tilde{\mathfrak{g}} \rightarrow T T(M / G)$ be the map defined by

$$
Z_{h}(\bar{X} \oplus \tilde{\xi})=T T \pi_{M / G} \bar{Z}\left[\bar{X}^{h}(x)+\xi_{L}^{V}(x)\right]_{G},
$$

where $\bar{X}^{h}$ is an invariant horizontal vector field that projects to $\bar{X}$ at $x \in M$, and $\xi_{L}^{V}$ is the left-invariant vertical vector field with value $\xi_{M}(x)$ at $x \in M$.

Let $Z_{v}: T(M / G) \oplus \tilde{\mathfrak{g}} \rightarrow T \tilde{\mathfrak{g}}$ be the map defined by

$$
Z_{v}(\bar{X} \oplus \tilde{\xi})=T \rho_{A} \bar{Z}\left(\left[\bar{X}^{h}(x)+\xi_{L}^{V}(x)\right]_{G}\right),
$$

where $\bar{X}^{h}$ and $\xi_{L}^{V}$ are defined as above. The following statements hold:

(i) $Z_{h}(\bar{X} \oplus \tilde{\xi})=S_{Z}(\bar{X})-\operatorname{vlft}_{\bar{X}} \mathscr{S}(\tilde{\xi}, \tilde{\xi})-\operatorname{vlft}_{\bar{X}}\left(T \pi_{M / G}\left\langle\bar{X}^{h}: \xi_{L}^{V}\right\rangle\right)$;

(ii) $Z_{v}(\tilde{X} \oplus \tilde{\xi})=R_{Z}(\bar{X})+U_{Z}(\tilde{\xi})-\operatorname{vlft}_{\xi}\left(\widetilde{\nabla}_{\bar{X}}^{A} \tilde{\xi}\left([x]_{G}\right)\right)$.

Proof. Let us compute

$$
\begin{aligned}
T T \pi_{M / G} Z & \left(\bar{X}^{h}(x)+\xi_{L}^{V}(x)\right) \\
= & T T \pi_{M / G}\left(\left(\bar{X}_{h}+\xi_{L}^{V}\right)^{T}\left(\bar{X}^{h}+\xi_{L}^{V}(x)\right)\right)-\operatorname{vlft}_{\bar{X}^{h}(x)}\left(T \pi_{M / G} \nabla_{\bar{X}^{h}+\xi_{L}^{V}}\left(X^{h}+\xi_{L}^{V}\right)\right) \\
= & T T \pi_{M / G}\left(\bar{X}^{h}\right)^{T}\left(\xi_{M}(x)\right)+\bar{X}^{T}\left(\bar{X}\left([x]_{G}\right)-\operatorname{vlft}_{\bar{X}^{h}(x)}\left(T \pi_{M / G} \nabla_{\bar{X}^{h}} \bar{X}^{h}\right)\right. \\
& -\operatorname{vlft}_{\bar{X}^{h}(x)}(\mathscr{S}(\tilde{\xi}, \tilde{\xi}))-\operatorname{vlft}_{\bar{X}^{h}(x)}\left(T \pi_{M / G}\left\langle\bar{X}^{h}: \xi_{L}^{V}\right\rangle\right) \\
= & T T \pi_{M / G}\left(\bar{X}^{h}\right)^{T}\left(\xi_{L}^{V}(x)\right)+S_{Z}(\bar{X})-\operatorname{vlft}_{\bar{X}^{h}(x)}(\mathscr{S}(\tilde{\xi}, \tilde{\xi})) \\
& -\operatorname{vlft}_{\bar{X}^{h}(x)}\left(T \pi_{M / G}\left\langle\bar{X}^{h}: \xi_{L}^{V}\right\rangle(x)\right) .
\end{aligned}
$$

We also have

$$
T T \pi_{M / G}\left(\bar{X}^{h}+\xi_{L}^{V}\right)^{T}\left(\xi_{L}^{V}(x)\right)=\left.\frac{d}{d t}\right|_{t=0} T \pi_{M / G} \xi_{L}^{V}\left(\Phi_{t}^{\bar{X}^{h}}(x)\right)=0 .
$$

This gives us the first part. Part (ii) follows from a similar computation. 
Remark 4.9. The fact that the right-hand sides of $Z_{h}$ and $Z_{v}$ respectively are independent of the extensions follows from $G$-invariance of $\omega$ and the definition of $\bar{Z}$.

Remark 4.10. The decomposition of the reduced geodesic spray into horizontal and vertical parts given in Theorem 4.8 is similar to the decomposition of second-order systems in Crampin and Mestdag [13], particularly in the case of an affine spray. We discuss the Riemannian case in Section 5.1.

\section{Discussion}

The horizontal part of the reduced geodesic spray consists of three terms. The map $S_{Z}$ is a second-order vector field on $T(M / G)$. The term $\mathscr{S}(\tilde{\xi}, \tilde{\xi})$ can be interpreted in the following manner. Recall that the second fundamental form corresponding to the vertical distribution is a map $\mathcal{S}: \Gamma(V M) \times \Gamma(V M) \rightarrow H M$ defined by

$$
\mathcal{S}\left(v_{x}, w_{x}\right)=\operatorname{hor}\left(\nabla_{X} Y\right), \quad v_{x}, w_{x} \in V_{x} M,
$$

where $X$ and $Y$ are extensions of $v_{x}$ and $w_{x}$ respectively. In view of this, we have

$$
\mathcal{S}\left(\xi_{M}(x), \xi_{M}(x)\right)=(\mathscr{S}(\tilde{\xi}, \tilde{\xi}))^{h}(x) .
$$

Now, the vertical distribution $V M$ is geodesically invariant if and only if $\mathcal{S}$ is skew-symmetric. Hence, if $V M$ is geodesically invariant, we have $\mathscr{S}(\tilde{\xi}, \tilde{\xi})=0$.

\subsection{The Riemannian case}

The last term in the horizontal part of the reduced geodesic spray is related to the curvature of the horizontal distribution, at least in the case when $M$ is a Riemannian manifold with an invariant Riemannian metric, the chosen affine connection is the Levi-Civita connection corresponding to this metric, and $A$ is the mechanical connection as we show below.

Let $(M, k)$ be a Riemannian manifold and $G$ be a Lie group that acts freely and properly on $G$, so that $\pi_{M / G}: M \rightarrow M / G$ is a principal bundle. Suppose that the Riemannian metric $k$ is invariant under $G$. The mechanical connection corresponding to $k$ is a principal connection on $\pi_{M / G}: M \rightarrow M / G$ determined by the condition that the horizontal subbundle is orthogonal to the vertical subbundle $V M$ with respect to the metric. We denote by $A$ the connection one-form corresponding to this connection. We also let $\nabla$ be the Levi-Civita connection corresponding to $k$.

Lemma 5.1. The following holds

$$
k\left(\left\langle\bar{X}^{h}: \xi_{L}^{V}\right\rangle(x), \bar{Y}^{h}(x)\right)=k\left(\left(B_{A}\left(\bar{X}^{h}(x), \bar{Y}^{h}(x)\right)\right)_{M}, \xi_{L}^{V}(x)\right),
$$

where $\bar{X}^{h}$ and $\bar{Y}^{h}$ are invariant horizontal vector fields on $M$, and $B_{A}$ is the curvature form corresponding to $A$.

Proof. Recall that if $X, Y$ and $Z$ are vector fields on $M$, the Koszul formula is given by

$$
\begin{aligned}
2 k\left(\nabla_{X} Y, Z\right)= & \mathscr{L}_{X}(k(Y, Z))+\mathscr{L}_{Y}(k(X, Z))-\mathscr{L}_{Z}(k(X, Y))+k([X, Y], Z) \\
& -k([X, Z], Y])-k([Y, Z], X) .
\end{aligned}
$$

We therefore have (using the Koszul formula twice and adding the two results)

$$
\begin{aligned}
& 2 k\left(\left\langle\bar{X}^{h}: \xi_{L}^{V}\right\rangle(x), \bar{Y}^{h}(x)\right)=2 \mathscr{L}_{\bar{X}^{h}}\left(k\left(\bar{Y}^{h}(x), \xi_{L}^{V}(x)\right)\right)+2 \mathscr{L}_{\xi_{L}^{V}}\left(k\left(\bar{X}^{h}(x), \bar{Y}^{h}(x)\right)\right) \\
& \quad-2 \mathscr{L}_{\bar{Y}^{h}}\left(k\left(\bar{X}^{h}(x), \xi_{L}^{V}(x)\right)\right)-2 k\left(\left[\bar{X}^{h}, \bar{Y}^{h}\right](x), \xi_{L}^{V}(x)\right)-2 k\left(\left[\xi_{L}^{V}, \bar{Y}^{h}\right](x), \bar{X}^{h}(x)\right) .
\end{aligned}
$$


Now, the first and the third terms respectively on the right-hand side are clearly zero (by the definition of the mechanical connection). The second term is zero since the function $k\left(\bar{x}^{h}(x), \bar{Y}^{h}(x)\right)$ is constant along the invariant vertical vector field $\xi_{L}^{V}$. The fifth term is also zero since the Lie bracket $\left[\xi_{L}^{V}, \bar{Y}^{h}\right]$ is a vertical vector field. Thus, we get

$$
k\left(\left\langle\bar{X}^{h}: \xi_{L}^{V}\right\rangle(x), \bar{Y}^{h}(x)\right)=k\left(\left[\bar{X}^{h}, \bar{Y}^{h}\right](x), \xi_{L}^{V}(x)\right) .
$$

By the Cartan structure formula, we have

$$
\left[\bar{X}^{h}, \bar{Y}^{h}\right]=[\bar{X}, \bar{Y}]^{h}-\left(B_{A}\left(\bar{X}^{h}, \bar{Y}^{h}\right)\right)_{M}(x) .
$$

Therefore,

$$
k\left(\left\langle\bar{X}^{h}: \xi_{L}^{V}\right\rangle(x), \bar{Y}^{h}(x)\right)=k\left(\left(B_{A}\left(\bar{X}^{h}(x), \bar{Y}^{h}(x)\right)\right)_{M}, \xi_{L}^{V}(x)\right) .
$$

The vertical part of the reduced geodesic spray consists of the map $R_{Z}$ which vanishes identically if the horizontal distribution corresponding to the principal connection $A$ is geodesically invariant, and can be thought of as the fundamental form corresponding to the horizontal distribution. Lewis [17] has shown that if both $H M$ and $V M$ are geodesically invariant, then the corresponding linear connection restricts to the subbundle $L(M, A)$. The term $U_{Z}(\tilde{\xi})$ is essentially the Euler-Poincaré term, and the last term corresponds to a connection on $\tilde{\mathfrak{g}}$.

\subsection{Forces in mechanics}

As mentioned in the Introduction, our motivation for studying reduction in the affine connection setup comes from mechanics. In this sense, studying the geodesic spray corresponds to looking at mechanical systems with no external forces (in other words, the dynamics are given by the geodesic equation). It is worth considering the case in which forces are present as many important examples in mechanics fall in this class. In the following, we consider the so-called simple mechanical systems $[1,5,21]$. A simple mechanical system is a triple $(M, k, V)$ where $(M, k)$ is Riemannian manifold and $V: M \rightarrow \mathbb{R}$ is a smooth function (called the potential function). The gradient of $V$ is a vector field on $M$ defined by

$$
\operatorname{grad} V(x)=k^{\#}(d V(x)),
$$

where $k^{\#}: T^{*} M \rightarrow T M$ is a vector bundle isomorphism over $M$ induced by the metric $k$. The dynamics of such a system are given by

$$
\nabla_{c^{\prime}(t)} c^{\prime}(t)=-(\operatorname{grad} V)(c(t)), \quad c(t) \in M,
$$

where $\nabla$ is the Levi-Civita connection on $M$. Equivalently, one can study the following equation on $T M[1]$ :

$$
v^{\prime}(t)=Z(v(t))-\operatorname{vlft}_{v(t)}(\operatorname{grad} V(c(t))), \quad v(t) \in T M, \quad \tau_{M}(v(t))=c(t) .
$$

In the unforced case dealt with in this paper, we study the geodesic spray because its integral curves project to geodesics on $M$. Similarly, we can study the second-order vector field

$$
\widetilde{Z}(v)=Z(v)-\operatorname{vlft}_{v}\left(\operatorname{grad} V\left(\tau_{M}(v)\right)\right),
$$

which, by Proposition 3.4, can be written as

$$
\widetilde{Z}(v)=X^{T}(v)-\operatorname{vlft}_{v}\left(\nabla_{X_{v}} X_{v}+\operatorname{grad} V\left(\tau_{M}(v)\right)\right) .
$$

If $\nabla, k$ and $V$ are $G$-invariant, we can study the reduction of $\widetilde{Z}$ using our methodology. Even though we consider a potential force here, a general force $F$ can be incorporated in this picture by essentially replacing $\operatorname{grad} V$ with $k^{\#}(F)$ in (5.1). 


\subsection{Generalized connections}

In our investigation, we have considered a $G$-invariant affine connection $\nabla$ on a manifold $M$ along with a principal connection $A$ on $\pi_{M / G}: M \rightarrow M / G$ and used it to define an $A$-dependent affine connection $\nabla^{A}$ on $M / G$. Equivalently, and perhaps more naturally, $\nabla$ induces a connection on the vector bundle $\left[\tau_{M}\right]_{G}: T M / G \rightarrow M / G$. Since the principal connection $A$ provides a decomposition of $T M / G$, we can also recover $\nabla^{A}$ in this manner.

Furthermore, we can consider a generalized connection $[8,11]$ on the vector bundle $\left[\tau_{M}\right]_{G}$ : $T M / G \rightarrow M / G$ and explore how our reduction procedure can be applied to this more general situation.

\subsection{Nonholonomic systems with symmetry}

Roughly speaking, nonholonomic systems are mechanical systems with velocities constrained to lie in a given non-integrable distribution. Following the fundamental paper of Koiller [16] there has been a lot of interest in studying symmetries and reduction of nonholonomic systems $[2,4,5,7,12,17,22]$. In this section we outline how these systems can be studied in our framework. Let $(M, k)$ be a Riemannian manifold with a $G$-invariant Riemannian metric. Let $\mathcal{D}$ be a smooth, non-integrable, $G$-invariant distribution on $M$ and $\mathcal{D}^{\perp}$ the orthogonal complement with respect to the metric $k$. Let $\nabla$ be the Levi-Civita affine connection associated with $k$. The Lagrange-d'Alembert principle allows us to conclude that the constrained geodesics $c(t) \in M$ satisfy [17]

$$
\nabla_{c^{\prime}(t)} c^{\prime}(t) \in \mathcal{D}_{c(t)}^{\perp}, \quad c^{\prime}(t) \in \mathcal{D}_{c(t)} .
$$

Sometimes these conditions are written as

$$
\nabla_{c^{\prime}(t)} c^{\prime}(t)=\lambda(c(t)), \quad P^{\perp}\left(c^{\prime}(t)\right)=0
$$

where $\lambda$ is a section of $\mathcal{D}^{\perp}$ and $P^{\perp}: T M \rightarrow T M$ is the projection onto $\mathcal{D}^{\perp}$. It can be shown that the trajectories $c: \mathbb{R} \rightarrow M$ satisfying the constraints are actually geodesics of an affine connection $\widetilde{\nabla}$ defined by $\widetilde{\nabla}_{X} Y=\nabla_{X} Y+\left(\nabla_{X} P^{\perp}\right)(Y)$. Note that, in general, the connection $\widetilde{\nabla}$ (sometimes called a constrained connection) will not be Levi-Civita. We can use our approach to study the geodesic spray of the constrained connection in the presence of a principal connection on $\pi_{M / G}: M \rightarrow M / G$. In such a case, the picture gets more complicated since a decomposition of $T T M / G$, and that of the geodesic spray, will depend on the distribution $\mathcal{D}$, and we hope to address this problem in subsequent work. This procedure is related to the reduction of "external" symmetries of a generalized $G$-Chaplygin system [14].

\section{Acknowledgements}

I would like to thank my thesis supervisor Dr. Andrew Lewis for his constant guidance and support. This work would not have materialized without the many invaluable discussions I have had with him over the years. The author also thanks the anonymous referees for their constructive comments on a previous version of this paper.

\section{References}

[1] Abraham R., Marsden J.E., Foundations of mechanics, 2nd ed., Benjamin/Cummings Publishing Co., Inc., Advanced Book Program, Reading, Mass., 1978.

[2] Bates L., Problems and progress in nonholonomic reduction, Rep. Math. Phys. 49 (2002), 143-149. 
[3] Bhand A., Geodesic reduction via frame bundle geometry, PhD Thesis, Queen's University, Kingston, ON, Canada, 2007, available at https://qspace.library.queensu.ca/handle/1974/456.

[4] Bloch A.M., Krishnaprasad P.S., Marsden J.E., Murray R.M., Nonholonomic mechanical systems with symmetry, Arch. Rational Mech. Anal. 136 (1996), 21-99.

[5] Bullo F., Lewis A.D., Geometric control of mechanical systems. Modeling, analysis, and design for simple mechanical control systems, Texts in Applied Mathematics, Vol. 49, Springer-Verlag, New York, 2005.

[6] Bullo F., Lewis A.D., Reduction, linearization, and stability of relative equilibria for mechanical systems on Riemannian manifolds, Acta Appl. Math. 99 (2007), 53-95.

[7] Cantrijn F., de León M., Marrero J.C., Martín de Diego D., Reduction of nonholonomic mechanical systems with symmetries, Rep. Math. Phys. 42 (1998), 25-45.

[8] Cantrijn F., Langerock B., Generalised connections over a vector bundle map, Differential Geom. Appl. 18 (2003), 295-317, math.DG/0201274.

[9] Cendra H., Marsden J.E., Ratiu T.S., Geometric mechanics, Lagrangian reduction, and nonholonomic systems, in Mathematics Unlimited-2001 and Beyond, Springer, Berlin, 2001, 221-273.

[10] Cendra H., Marsden J.E., Ratiu T.S., Lagrangian reduction by stages, Mem. Amer. Math. Soc. 152 (2001), no. 722, 108 pages.

[11] Cortés J., Martínez E., Mechanical control systems on Lie algebroids, IMA J. Math. Control Inform. 21 (2004), 457-492.

[12] Cortés Monforte J., Geometric, control and numerical aspects of nonholonomic systems, Lecture Notes in Mathematics, Vol. 1793, Springer-Verlag, Berlin, 2002.

[13] Crampin M., Mestdag T., Reduction and reconstruction aspects of second-order dynamical systems with symmetry, Acta Appl. Math. 105 (2009), 241-266, arXiv:0807.0156.

[14] Ehlers K., Koiller J., Montgomery R., Rios P.M., Nonholonomic systems via moving frames: Cartan equivalence and Chaplygin Hamiltonization, in The Breadth of Symplectic and Poisson Geometry, Progr. Math., Vol. 232, Birkhäuser Boston, Boston, MA, 2005, 75-120, math-ph/0408005.

[15] Kobayashi S., Nomizu K., Foundations of differential geometry, Tracts in Pure and Applied Mathematics, Vol. 1, Interscience Publishers, New York - London, 1964.

[16] Koiller J., Reduction of some classical nonholonomic systems with symmetry, Arch. Rational Mech. Anal. 118 (1992), 113-148.

[17] Lewis A.D., Affine connections and distributions with applications to nonholonomic mechanics, Rep. Math. Phys. 42 (1998), 135-164.

[18] Lewis A.D., Murray R.M., Decompositions for control systems on manifolds with an affine connection, Systems Control Lett. 31 (1997), 199-205.

[19] Marsden J.E., Ratiu T.S., Introduction to mechanics and symmetry. A basic exposition of classical mechanical systems, 2nd ed., Texts in Applied Mathematics, Vol. 17, Springer-Verlag, New York, 1999.

[20] Marsden J.E., Weinstein A., Reduction of symplectic manifolds with symmetry, Rep. Math. Phys. 5 (1974), 121-130.

[21] Smale S., Topology and mechanics, Invent. Math. 10 (1970), 305-331.

[22] Śniatycki J., Nonholonomic Noether theorem and reduction of symmetries, Rep. Math. Phys. 42 (1998), $5-23$. 